\title{
Influência do modo de aplicação de carga nas propriedades de fratura de uma mistura asfáltica de agregados finos
}

\author{
Gustavo Adolfo Badilla Vargas ${ }^{1}$, Alex Duarte de Oliveira ${ }^{2}$, Francisco Thiago Sacramento Aragão ${ }^{3}$ \\ ${ }^{1}$ Programa de Engenharia Civil (COPPE) - Universidade Federal do Rio de Janeiro, gustavo.badilla@coc.ufrj.br \\ 2Programa de Engenharia Civil (COPPE) - Universidade Federal do Rio de Janeiro, alexd@coc.ufrj.br \\ ${ }^{3}$ Programa de Engenharia Civil (COPPE) - Universidade Federal do Rio de Janeiro, fthiago@coc.ufrj.br
}

\section{Recebido:}

6 de março de 2018

Aceito para publicação:

24 de agosto de 2018

Publicado:

4 de novembro de 2018

Editor de área:

Kamilla Vasconcelos

\section{Palavras-chaves:}

Misturas asfálticas,

Propriedades de fratura,

Modelo de zona coesiva,

Simulações numéricas.

\section{Keywords:}

Asphalt mixtures,

Fracture properties,

Cohesive zone model,

Numerical simulations.

DOI:10.14295/transportes.v26i3.1609

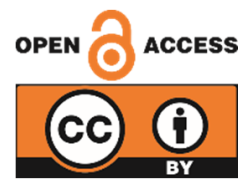

\begin{abstract}
RESUMO
Entre os diferentes modos de dano que comprometem a vida útil de misturas asfálticas, o trincamento é, provavelmente, um dos mais complexos e de difícil caracterização, uma vez que a resistência à fratura destes materiais é altamente dependente da temperatura e das condições de carregamento. Neste estudo investigou-se a influência das condições de carregamento sobre os parâmetros de fratura de uma mistura asfáltica de agregados finos, determinados a partir de simulações de ensaios de flexão em semicírculos (semicircular bending - SCB). As simulações de SCB foram realizadas por meio do método dos elementos finitos e do modelo bilinear de zona coesiva, considerando apenas o modo I de fratura e três diferentes formas de carregamento. Os resultados obtidos (energia de fratura, resistência coesiva e curvas Força-CTOD) demonstraram que a forma de representar o carregamento exerce influência significativa sobre os parâmetros de fratura e, consequentemente, sobre a aplicabilidade deste tipo de procedimento numérico-experimental.
\end{abstract}

\begin{abstract}
Among the different distresses that compromise the service life of asphalt mixtures, cracking is probably one of the most complexes and hard to be predicted. The cracking resistance of such materials is highly dependent of temperature and loading conditions. In this study, it was investigated the effects of loading conditions on the fracture parameters of a fine aggregate asphalt mixture, determined from simulations of semi-circular bending $(\mathrm{SCB})$ tests. The SCB simulations were performed using the finite element method and the bilinear cohesive zone model, considering only the fracture mode I and three different loading configurations. The results (fracture energy, cohesive strength and Force-CTOD curves) demonstrated that the loading configuration exerts a significant influence on the fracture parameters and, consequently, on the applicability of this type of numerical-experimental procedure.
\end{abstract}

\section{INTRODUÇÃO}

Na literatura, encontram-se diversos protocolos de ensaio, com diferentes configurações de geometria e carregamento, desenvolvidos para caracterizar as propriedades de fratura de misturas asfálticas. Entre estes protocolos, os mais utilizados são: o ensaio de flexão em viga com uma fenda (single-edge notched beam - SEB), o ensaio de tração em disco com fenda (disk-shaped compaction tension test - DCT) e o ensaio de flexão em semicírculos com fenda (semi-circular bending - SCB) (Marasteanu et al., 2002; Wagoner et al., 2005; Song et al., 2008; Hirsch, 2009; Budny, 2012; Li e Marasteanu, 2012; Marasteanu et al., 2012). 
De acordo com Aragão et al. (2014), os ensaios SCB apresentam algumas vantagens com relação aos outros tipos de ensaios de fratura, tais como: (1) a quantidade de corpos de prova que podem ser obtidos a partir de uma única amostra cilíndrica de mistura asfáltica compactada; (2) a facilidade de obtenção de amostras de campo e (3) a simplicidade dos procedimentos experimentais. Os autores apontaram como desvantagem desta configuração o tamanho reduzido da área potencial de fratura.

Em paralelo aos protocolos experimentais, alguns pesquisadores também desenvolveram modelos numéricos computacionais que incorporam as propriedades obtidas nos diversos ensaios para tentar prever o comportamento de misturas asfálticas na fratura. Os modelos computacionais são utilizados, atualmente, por oferecerem muitos recursos e serem facilmente ajustados, possibilitando a representação de diversos problemas reais complexos e, consequentemente, possuírem grande aplicabilidade.

Apesar de cada vez mais robustos e realistas, estes modelos computacionais precisam ser sempre calibrados e validados a partir da comparação das suas previsões com os resultados de ensaios de laboratório. Uma técnica usada com frequência em simulações de ensaios de trincamento tem sido a análise inversa, para a obtenção das propriedades de fratura dos materiais. Neste tipo de procedimento, parâmetros globais como força e deslocamento são monitorados nos ensaios das amostras virtuais e comparados com aqueles obtidos em ensaios de laboratório correspondentes. As propriedades de fratura são ajustadas de maneira iterativa até que se observe equivalência entre os dois conjuntos de dados. Como exemplo, Aragão et al. (2014) propuseram um procedimento deste tipo para a obtenção de propriedades de fratura de misturas asfálticas, utilizando o método dos elementos finitos, o modelo bilinear de zona coesiva e resultados de ensaios de SCB.

Os modelos computacionais são ferramentas muito importantes para o estudo do comportamento dos materiais. No entanto, é importante ressaltar que a aplicação destes métodos para prever fenômenos complexos, como o processo de fratura em misturas asfálticas, pode fornecer resultados inconsistentes, caso o problema físico não seja representado corretamente ou os métodos não sejam aplicados de maneira adequada.

Em geral, o sucesso das previsões feitas por modelos computacionais depende da seleção adequada dos modelos constitutivos e de resistência ao dano, para representar de maneira realista as respostas dos materiais ao carregamento. Além disso, deve-se atentar para a correta representação de condições de contorno nos ensaios virtuais. Nesse contexto, o presente estudo avaliou a influência que o modo de representação numérica do carregamento exerce sobre a determinação dos parâmetros de fratura de misturas asfálticas de agregados finos, determinados seguindo o procedimento de análise inversa proposto por Aragão et al. (2014).

Este trabalho é parte fundamental de um modelo computacional que está sendo desenvolvido para prever o comportamento mecânico microestrutural de misturas asfálticas. Este estudo investigou apenas o comportamento da MAF, mas, nas etapas seguintes da pesquisa, será estudado o comportamento do concreto asfáltico. Espera-se que a versão final do modelo se torne uma ferramenta eficiente de análise de pavimentos asfálticos, com a qual previsões sobre o comportamento mecânico das misturas poderão ser feitas, a partir do conhecimento das propriedades dos seus constituintes, adequadamente caracterizadas, e da definição correta das condições de contorno da estrutura do pavimento. Em outras palavras, espera-se que ensaios virtuais possam resultar na otimização dos programas experimentais, gerando significativa economia de tempo e recursos. 


\section{MATERIAIS E PREPARAÇÃO DE AMOSTRAS}

A MAF usada neste trabalho foi obtida a partir de um ligante asfáltico classificado pela metodologia Superpave como PG 64-22 S, uma mistura de agregados finos passando na peneira \#16 (abertura de malha de 1,18 mm) e cal hidratada CH-I. A granulometria adotada para a fabricação da MAF é apresentada na Figura 1, que também mostra a granulometria do Concreto Betuminoso Usinado a Quente (CBUQ) correspondente à MAF.

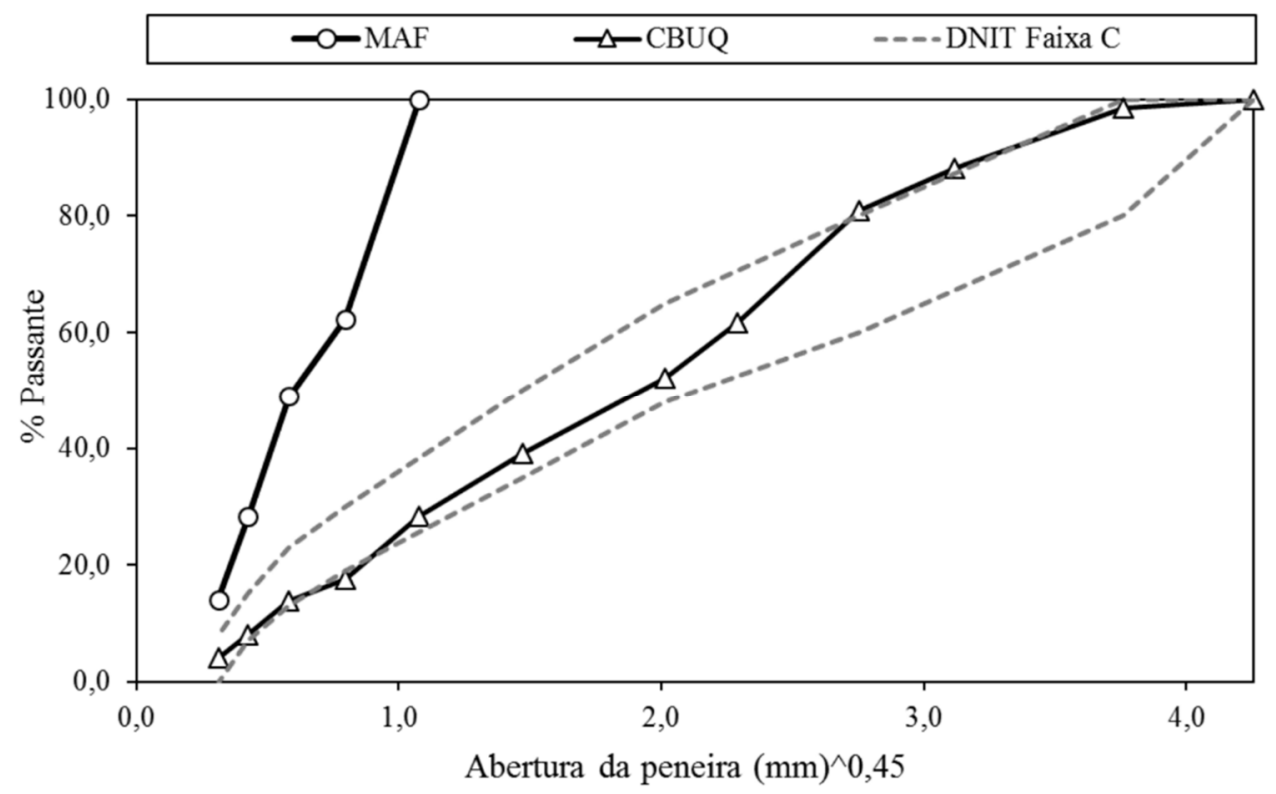

Figura 1: Granulometria da MAF correspondente à mistura CBUQ dosada neste trabalho.

A dosagem da MAF foi baseada nas características volumétricas do CBUQ, de acordo com o método de dosagem de MAF proposto por Sousa et al. (2013), resultando em um teor de ligante de 8,8\%. Os agregados constituintes da MAF representaram 28,4\% da massa total de agregados do CBUQ. Foi usado 3,5\% de cal em relação à massa total de agregados da MAF. Durante o processo de dosagem, as proporções dos agregados e do fíler que compõem a MAF foram mantidas iguais às usadas na composição da mistura asfáltico correspondente. As amostras foram moldadas com um volume de vazios de $4 \%$.

A Figura 2 apresenta um resumo do procedimento numérico-experimental adotado. Amostras de MAF medindo $150 \mathrm{~mm}$ em diâmetro foram fabricadas usando um compactador giratório da metodologia Superpave (CGS). Foram extraídos dois tipos de corpos de provas: (a) amostras cilíndricas medindo $12 \mathrm{~mm}$ em diâmetro e $50 \mathrm{~mm}$ em altura (bastonetes) e (b) amostras SCB medindo $150 \mathrm{~mm}$ em diâmetro, $75 \mathrm{~mm}$ em altura e $25 \mathrm{~mm}$ em espessura. Uma fenda foi cortada em cada amostra SCB, próximo à linha de simetria. 0 tamanho das fendas foi ajustado para gerar ligamentos de $50 \mathrm{~mm}$ nas amostras SCB.

Com as amostras cilíndricas de $12 \mathrm{~mm}$ em diâmetro e $50 \mathrm{~mm}$ em altura, foram determinadas propriedades viscoelásticas lineares da MAF, em um reômetro de cisalhamento dinâmico (dynamic shear rheometer - DSR). Para as amostras SCB, foram conduzidos testes de fratura com diferentes temperaturas $\left(-10^{\circ} \mathrm{C}, 10^{\circ} \mathrm{C}\right.$ e $\left.25^{\circ} \mathrm{C}\right)$ e taxas de propagação de trincas $(0,5 \mathrm{~mm} / \mathrm{min}$., 1,0 $\mathrm{mm} / \mathrm{min}$. e 2,0 $\mathrm{mm} / \mathrm{min}$.). Os resultados obtidos dos ensaios de laboratório foram utilizados como dados de entrada para a realização das simulações numéricas. 


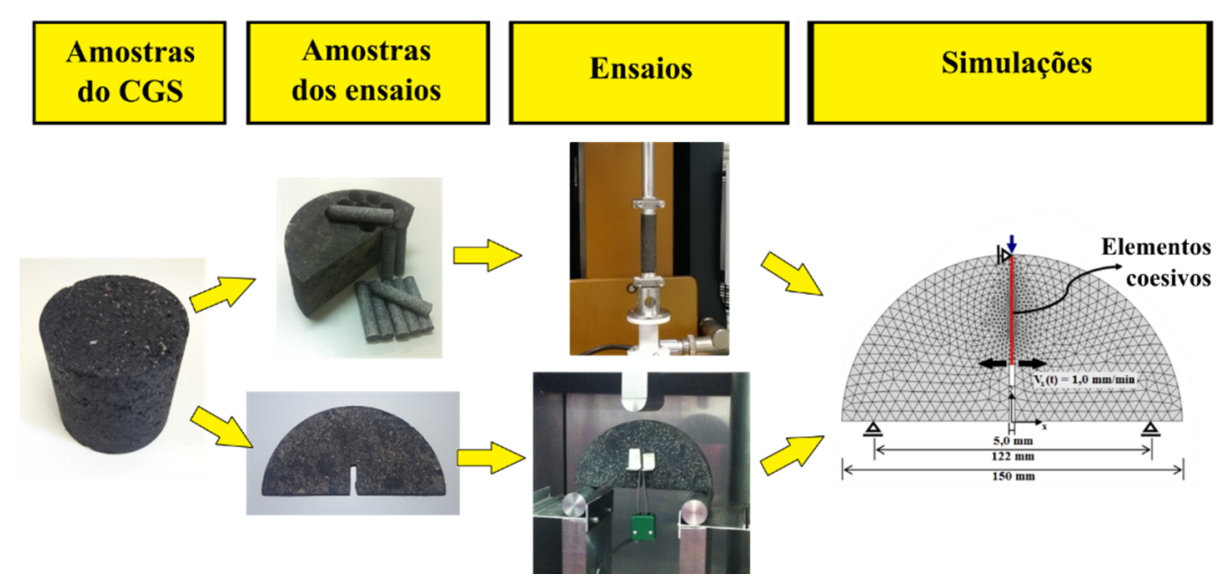

Figura 2: Resumo do procedimento numérico-experimental adotado neste trabalho.

\subsection{Propriedades viscoelásticas lineares da MAF}

As amostras usadas para a caracterização de propriedades viscoelásticas lineares de MAF foram produzidas em um CGS com volume de vazios entre 4\% e 5\% e mediram $150 \mathrm{~mm}$ em diâmetro e $90 \mathrm{~mm}$ em altura. Em seguida, foi necessário serrar o topo e a base das amostras para produzir cilindros com $150 \mathrm{~mm}$ de diâmetro e $50 \mathrm{~mm}$ de altura.

Para possibilitar a extração das amostras cilíndricas ( $12 \mathrm{~mm}$ de diâmetro e $50 \mathrm{~mm}$ de altura) de corpos de prova do CGS, foram necessárias: a fabricação e a adaptação de brocas especiais; o ajuste da velocidade de descida da broca; e, durante a extração, a aplicação contínua de água fria para evitar o aquecimento excessivo das amostras e a consequente alteração das suas características geométricas.

Durante as varreduras de frequência, as amostras foram ensaiadas nas temperaturas $5^{\circ} \mathrm{C}$, $15^{\circ} \mathrm{C}, 25^{\circ} \mathrm{C}, 35^{\circ} \mathrm{C}$ e $50^{\circ} \mathrm{C}$, com frequências variando entre $0,01 \mathrm{~Hz}$ e $25 \mathrm{~Hz}$. 0 princípio da superposição tempo-temperatura foi adotado para gerar curvas mestras de módulo de cisalhamento dinâmico, que é definido como a razão entre as amplitudes de tensão e deformação cíclicas.

A partir das curvas mestras obtidas, o método da colocação foi adotado para ajustar os coeficientes da função analítica em forma de série (série de Prony) do módulo cisalhante de armazenamento, com base no modelo generalizado de Maxwell, como indicado na Equação 1. Estes coeficientes foram posteriormente usados como parâmetros de entrada no modelo computacional para a simulação do comportamento viscoelástico linear da MAF.

$$
G(\omega)=G_{\infty}+\sum_{i=1}^{n} \frac{G_{i} \omega^{2} \rho_{i}^{2}}{\omega^{2} \rho_{i}^{2}+1}
$$

Onde:

$G^{\prime}(\omega)$ = módulo cisalhante de armazenamento;

$\omega=$ frequência angular;

$G_{\infty}=$ módulo de equilíbrio a um tempo longo de carregamento;

$G_{i}=$ constantes de mola do modelo generalizado de Maxwell;

$\rho_{i}=$ tempo de relaxação;

$n$ = número de unidades de Maxwell no modelo generalizado de Maxwell.

A Tabela 1 mostra os coeficientes da série de Prony obtidos segundo a Equação 1 e usados posteriormente como parâmetros de entrada na modelagem computacional para cada uma das 
temperaturas dos ensaios de fratura.

Tabela 1: Propriedades viscoelásticas lineares da MAF.

\begin{tabular}{|c|c|c|c|c|c|c|}
\hline Temperatura de referência & $-10 \div C$ & & $10 \div \mathrm{C}$ & & $25 \div \mathrm{C}$ & \\
\hline Parâmetros de série de Prony & $\rho_{\mathrm{i}}(\mathrm{s})$ & $\mathrm{Gi}(\mathrm{Pa})$ & $\rho_{\mathrm{i}}(\mathrm{s})$ & $\mathrm{Gi}(\mathrm{Pa})$ & $\rho_{\mathrm{i}}(\mathrm{s})$ & $\mathrm{Gi}(\mathrm{Pa})$ \\
\hline 1 & $5,54 \mathrm{E}-01$ & $1,82 \mathrm{E}+06$ & $4,00 \mathrm{E}-03$ & $2,01 E+08$ & $2,30 \mathrm{E}-04$ & $6,14 \mathrm{E}+07$ \\
\hline 2 & $5,54 \mathrm{E}+00$ & $7,32 E+08$ & $4,00 \mathrm{E}-02$ & $2,83 E+08$ & $2,30 \mathrm{E}-03$ & $5,52 E+08$ \\
\hline 3 & $5,54 \mathrm{E}+01$ & $7,92 E+08$ & $4,00 E-01$ & $8,21 E+08$ & $2,30 \mathrm{E}-02$ & $7,71 \mathrm{E}+08$ \\
\hline 4 & $5,54 \mathrm{E}+02$ & $4,06 E+08$ & $4,00 E+00$ & $5,62 E+08$ & $2,30 \mathrm{E}-01$ & $4,10 E+08$ \\
\hline 5 & $5,54 \mathrm{E}+03$ & $1,55 E+08$ & $4,00 E+01$ & $2,15 E+08$ & $2,30 E+00$ & $1,83 E+08$ \\
\hline 6 & $5,54 \mathrm{E}+04$ & $4,39 E+07$ & $4,00 \mathrm{E}+02$ & $7,48 \mathrm{E}+07$ & $2,30 \mathrm{E}+01$ & $1,04 \mathrm{E}+08$ \\
\hline 7 & $5,54 \mathrm{E}+05$ & $2,64 \mathrm{E}+07$ & $4,00 E+03$ & $2,53 E+07$ & $2,30 E+02$ & $2,69 E+07$ \\
\hline 8 & $5,54 \mathrm{E}+06$ & $9,58 E+06$ & $4,00 E+04$ & $1,28 \mathrm{E}+07$ & $2,30 E+03$ & $1,27 E+07$ \\
\hline 9 & $5,54 \mathrm{E}+07$ & $6,57 E+06$ & $4,00 E+05$ & $9,17 E+06$ & $2,30 E+04$ & $9,16 E+06$ \\
\hline$\infty$ & - & $2,22 \mathrm{E}+07$ & - & $2,22 \mathrm{E}+07$ & - & $2,22 \mathrm{E}+07$ \\
\hline
\end{tabular}

\subsection{Propriedades de fratura da MAF}

Antes de ensaiados, os corpos de prova foram condicionados em uma câmara até que a temperatura interna estabilizasse na de ensaio. Para este controle, uma amostra de sacrifício foi usada e sua temperatura interna foi medida com um termopar.

Seguindo a etapa de condicionamento, carregamentos foram aplicados às amostras pelo atuador de uma máquina universal MTS. Um sensor do tipo clip-on gauge foi montado na face frontal dos corpos de prova para monitorar a evolução da abertura da fenda inicial, na sua extremidade interna (crack tip opening displacement - CTOD). Este sensor monitorou a velocidade de abertura da trinca, que foi mantida constante a partir de um procedimento iterativo de ajuste do carregamento imposto pelo atuador da máquina, conhecido como feedback loop ou servocontrole. Um segundo clip-on gauge também foi instalado na extremidade externa da fenda inicial para o monitoramento do chamado crack mouth opening displacement (CMOD).

A escolha do CTOD (e não do CMOD) para o controle da taxa de abertura das trincas baseiase em pesquisas anteriores que demonstraram que em ensaios de fratura com taxas constantes de evolução do CMOD, as correspondentes taxas de evolução do CTOD não são constantes até que se atinja o pico de força (SONG et al., 2008). Isto pode indicar que a taxa de evolução do CTOD só se torna constante quando há a formação de macrofissuras a partir da coalescência de microfissuras. Assim, neste trabalho, foram impostas taxas constantes de abertura do CTOD, para garantir que todas as amostras fossem submetidas às mesmas taxas de propagação de trincas durante toda a duração dos ensaios. As taxas usadas foram $0,5 \mathrm{~mm} / \mathrm{min}, 1,0 \mathrm{~mm} / \mathrm{min}$ e 2,0 $\mathrm{mm} / \mathrm{min}$.

\subsection{Modelagem computacional para a obtenção de propriedades de fratura}

O comportamento viscoelástico da MAF cria uma complicação adicional na identificação de suas propriedades de fratura. De acordo com Aragão et al. (2014), a análise inversa para obter parâmetros de fratura a partir da comparação entre resultados experimentais e simulações numéricas é uma abordagem mais apropriada do que a suposição feita por abordagens tradicionais de equivalência entre propriedades da zona de processo de fratura e parâmetros de fratura, calculados com base em deformações médias de amostras em processo de fissuramento.

Um estudo feito por Aragão e Kim (2012) indicou, por exemplo, que parte da energia obtida a partir do cálculo da área sob curvas experimentais força-deslocamento está relacionada com a energia dissipada devido ao comportamento viscoelástico da matriz. Assim, os parâmetros de 
fratura ao longo da zona de processo de fratura devem ser identificados localmente e não a partir de resultados globais de força-deslocamento. Com base neste fato, a metodologia proposta por Aragão et al. (2014), que combina resultados de simulação numérica e oriundos de ensaios de laboratório, foi adotada nesta pesquisa.

A modelagem de fratura em misturas MAF foi baseada no método dos elementos finitos e no conceito de zonas coesivas de fratura. Amostras virtuais foram fabricadas e discretizadas para a geração da malha de elementos finitos. Um estudo de convergência do nível de refinamento das malhas foi conduzido para minimizar os erros de aproximação intrínsecos a métodos numéricos, como é o método dos elementos finitos. A Figura 3 apresenta a malha de elementos finitos, o modelo de zona coesiva bilinear e as condições de contorno adotadas para as simulações.

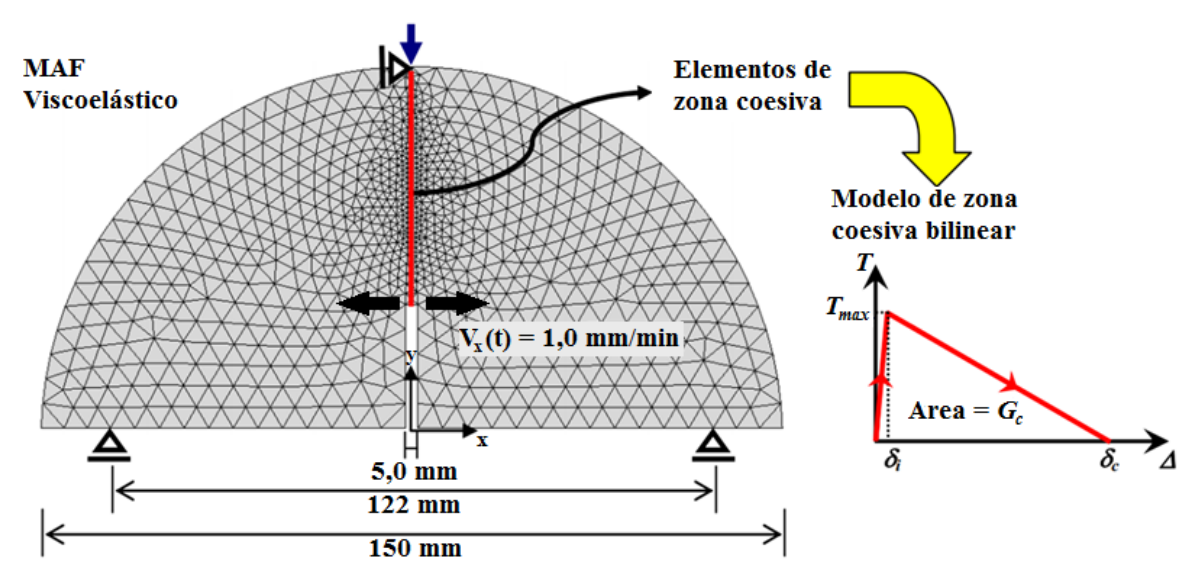

Figura 3: Malhas de elementos finitos usadas nas simulações numéricas e lei de tração-separação da zona coesiva para ensaios SCB para a taxa de $1 \mathrm{~mm} / \mathrm{min}$.

Durante as simulações, a resistência coesiva $\left(T_{\max }\right)$ e a energia de fratura $\left(G_{c}\right)$ foram ajustadas por um processo de tentativa e erro. Inicialmente, a resistência coesiva foi ajustada até a observação de equivalência entre os picos de força obtidos das simulações e dos correspondentes ensaios de fratura SCB conduzidos em diferentes temperaturas e taxas de abertura de CTOD. Depois disso, o outro parâmetro necessário para a definição do modelo coesivo bilinear, ou seja, a energia de fratura, foi ajustado até que fosse observada a equivalência entre os resultados numéricos e experimentais da região pós-pico das curvas força-tempo. 0 processo de calibração foi considerado completo quando os resultados da simulação ficaram próximos dos resultados experimentais. 0 critério utilizado para a calibração foi a minimização do somatório dos erros quadrados ( $\Sigma$ erro $\left.^{2}\right)$.

Para permitir a avaliação objetiva do nível de semelhança entre as curvas, foram adotados um procedimento de ajuste dos dados e um critério numérico de avaliação. 0 procedimento de ajuste dos dados consiste em representar as curvas força - deslocamento usando a série exponencial indicada na Equação 2. Esta representação matemática permite que a comparação entre as curvas possa ser feita para pontos específicos, o que facilita o cálculo de parâmetros estatísticos.

$F=$ força axial;

$$
F=\sum_{i=1}^{n} a_{i} e^{b_{i} C T O D}
$$


$a_{i}, b_{i}=$ constantes matemáticas no modelo;

$n=$ número de termos na série ( $\mathrm{n}=8$ valor adotado).

A Figura 4 ilustra um exemplo da aplicação do ajuste matemático para os resultados dos ensaios experimentais, indicando que o modelo matemático adotado foi capaz de representar adequadamente os dados. Os ajustes foram feitos usando o método dos mínimos quadrados e as constantes $a_{i}$ e $b_{i}$ de cada ajuste foram obtidas a partir de um processo iterativo de minimização de erros com a ferramenta de solver.

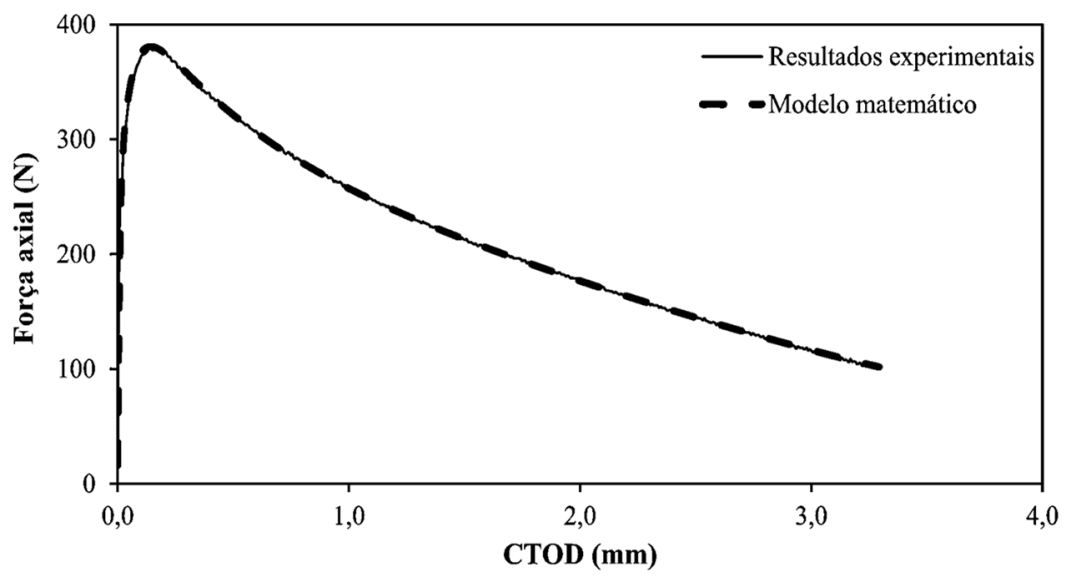

Figura 4: Exemplo de ajuste de uma expressão matemática para representar a curva força - deslocamento do ensaio de laboratório realizado para uma temperatura de $25^{\circ} \mathrm{C}$ e taxa de abertura do CTOD de $2,0 \mathrm{~mm} / \mathrm{min}$.

Para avaliar a semelhança entre as curvas, foram adotados três parâmetros estatísticos: (a) a raiz quadrada do erro médio (Root Mean Square Error - RMSE), posteriormente normalizada pelo valor da força pico; (b) a relação percentual entre os valores de força pico experimentais e numéricos; e (c) a relação percentual entre as áreas abaixo das curvas força - CTOD experimentais e numéricas.

Para cada um dos três parâmetros, foi definido como critério de aceitação que a diferença entre os valores referentes aos dados experimentais e numéricos fosse inferior a 15\%. Foram consideradas como válidas as simulações para as quais dois dos três parâmetros atenderam ao critério de aceitação. Considerando a complexidade do problema em estudo e que frequentemente réplicas de ensaios de fratura realizados em laboratório apresentam níveis de variabilidade semelhantes ou maiores do que estes, os autores consideram que uma tolerância de 15\% é aceitável.

\section{MODELAGEM COMPUTACIONAL}

Como já mencionado, para garantir a aplicação de uma taxa de abertura constante no CTOD durante os ensaios de fratura, foi necessário desenvolver um procedimento iterativo de ajuste do carregamento imposto pelo atuador da máquina do tipo feedback loop. Um procedimento semelhante foi implementado em forma de sub-rotina para permitir a simulação mais realista dos ensaios de fratura no Abaqus.

Foram realizadas simulações de SCB usando esta sub-rotina e simulações adicionais, sem o uso da sub-rotina e, consequentemente, sem o controle da taxa de CTOD. Estas simulações adicionais foram realizadas com controle apenas na taxa de deslocamento vertical do atuador de carga, localizado no topo da amostra. Propriedades de fratura foram determinadas para ensaios 
de fratura realizados nas temperaturas de $-10^{\circ} \mathrm{C}, 10^{\circ} \mathrm{C}$ e $25^{\circ} \mathrm{C}$ e com taxas de carregamento de $0,5 \mathrm{~mm} / \mathrm{min}$., $1,0 \mathrm{~mm} / \mathrm{min}$. e $2,0 \mathrm{~mm} / \mathrm{min}$.

A Figura 5 mostra os resultados das simulações conduzidas com os dois tipos de controle, para a MAF a $25^{\circ} \mathrm{C}$. Como observado, as simulações realizadas sem o uso da sub-rotina resultaram em taxas variáveis de abertura do CTOD. Já as simulações realizadas com a sub-rotina permitiram que taxas constantes de abertura do CTOD fossem aplicadas.

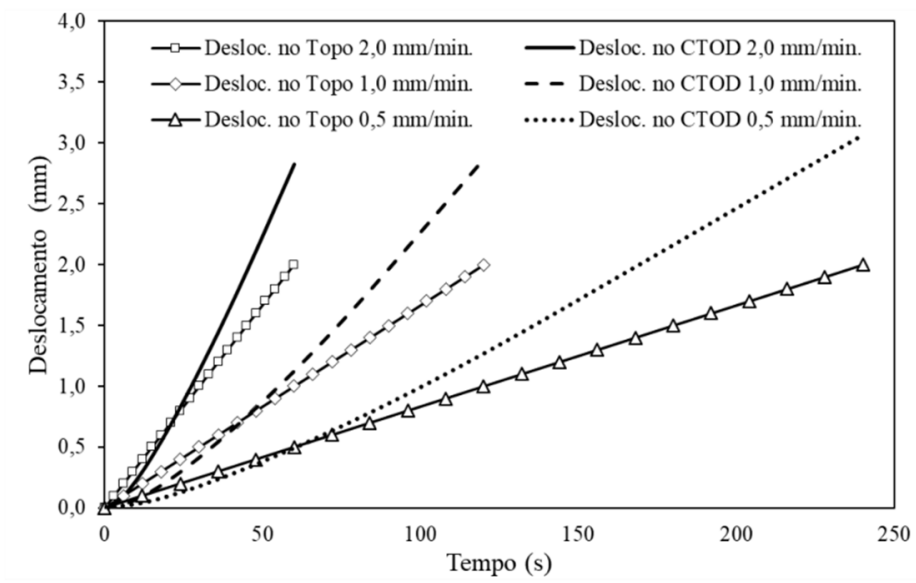

Figura 5: Variação dos deslocamentos do topo e da abertura do CTOD a $25^{\circ} \mathrm{C}$.

Além de permitir a utilização de sub-rotinas, o Abaqus também permite que funções matemáticas sejam definidas para representar o carregamento nas simulações. Desta forma, para tentar reproduzir as mesmas condições de contorno impostas às amostras durante os ensaios reais, foram realizadas simulações com controle da taxa de CTOD, sendo obtidas destas simulações as curvas de variação de deslocamento no topo das amostras como respostas das simulações com taxa constante de CTOD. Estas curvas de deslocamento foram ajustadas matematicamente, usando o mesmo tipo de série exponencial apresentado na Equação 2, para obter funções de deslocamento do topo das amostras, que foram inseridas no Abaqus, posteriormente, para tentar realizar simulações com controle indireto da taxa de abertura do CTOD. Este procedimento foi denominado de controle no topo.

Resultados de simulações considerando estes dois tipos de carregamento foram comparados com resultados experimentais, usando a mesma metodologia descrita anteriormente e adotando os mesmos critérios de aceitação. Os parâmetros estatísticos de comparação para as simulações feitas com a função de deslocamento do topo, tentando controlar indiretamente a abertura do CTOD, estão apresentados na Tabela 2. Os dados da tabela mostram que todas as simulações atenderam aos critérios de aceitação definidos no fim da seção 2.

Tabela 2: Parâmetros estatísticos obtidos para as simulações da MAF.

\begin{tabular}{llllllll}
\hline $\begin{array}{l}\text { Temperatura } \\
(\mathbf{0}-)\end{array}$ & $\begin{array}{l}\text { Taxa de } \\
\text { deslocamento } \\
\text { (mm/min) }\end{array}$ & CTOD $(\mathbf{m m}$.) & $\begin{array}{l}\text { RSME } \\
\text { (N) }\end{array}$ & $\mathbf{R}^{\mathbf{2}}$ & $\begin{array}{l}\text { Erro do } \\
\text { RSME }\end{array}$ & $\begin{array}{l}\text { Erro da } \\
\text { Área }\end{array}$ & $\begin{array}{l}\text { Erro da } \\
\text { Força Pico }\end{array}$ \\
\hline \multirow{3}{*}{25} & 2,0 & 4,0 & 38,5 & 0,95 & $10,6 \%$ & $0,1 \%$ & $3,2 \%$ \\
& 1,0 & 2,0 & 22,7 & 0,94 & $8,3 \%$ & $5,1 \%$ & $-8,8 \%$ \\
\hline \multirow{2}{*}{10} & 0,5 & 2,0 & 15,3 & 0,96 & $8,8 \%$ & $8,6 \%$ & $-5,6 \%$ \\
& 2,0 & 2,0 & 210,7 & 0,93 & $12,3 \%$ & $2,3 \%$ & $3,3 \%$ \\
& 1,0 & 2,0 & 260,2 & 0,87 & $14,3 \%$ & $-12,1 \%$ & $-10,6 \%$ \\
\hline
\end{tabular}




\begin{tabular}{cccccccc}
\hline \multirow{3}{*}{-10} & 2,0 & 0,3 & 184,5 & 0,97 & $8,8 \%$ & $-3,1 \%$ & $-1,4 \%$ \\
& 1,0 & 0,3 & 156,9 & 0,97 & $7,9 \%$ & $-8,2 \%$ & $1,5 \%$ \\
& 0,5 & 0,3 & 129,4 & 0,98 & $6,8 \%$ & $-4,9 \%$ & $-5,0 \%$ \\
\hline
\end{tabular}

A Figura 6 apresenta algumas comparações entre os resultados experimentais e os resultados das simulações realizadas no Abaqus com o controle no CTOD e com o controle no topo. Os gráficos da figura mostram que, para ambas as formas de controle, foi possível simular as condições de ensaio, obtendo curvas força-CTOD bastante próximas das curvas dos ensaios.
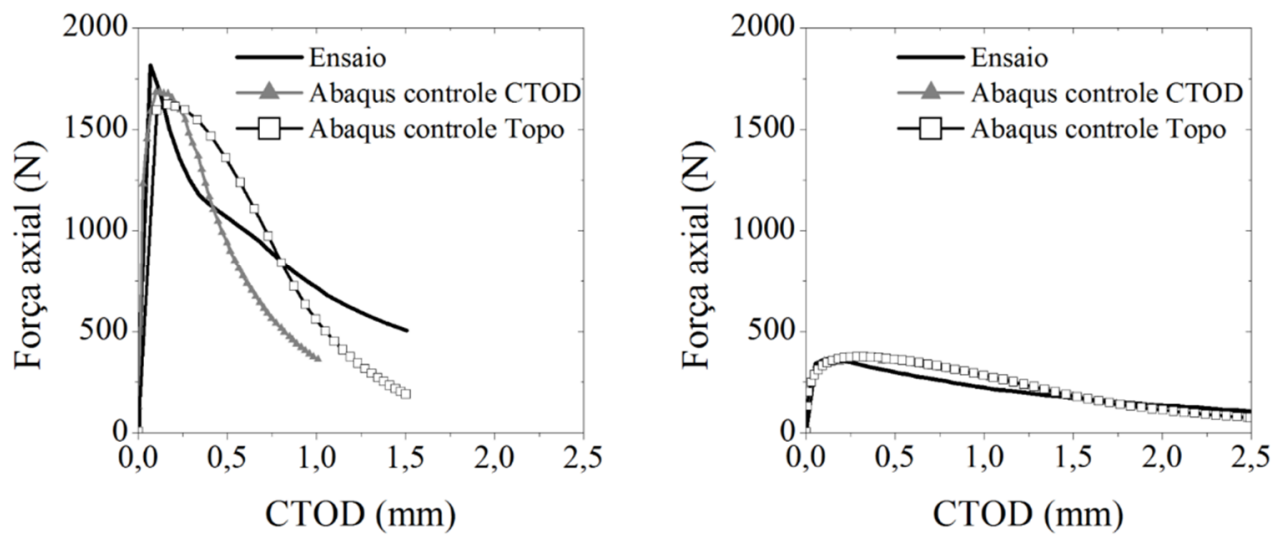

Figura 6. Comparações entre os resultados experimentais e os resultados das simulações realizadas com os dois modos de controle de carregamento.

Apesar da boa correlação entre as curvas experimentais e numéricas, é importante ressaltar que não foram utilizadas as mesmas propriedades de fratura nas simulações dos dois modos de controle, o que pode ser visto na Figura 7. No entanto, a Figura 7 indica que ambos permitem a obtenção de propriedades de fratura que variam com a temperatura e com a velocidade de propagação da fissura, que é o comportamento esperado para misturas asfálticas. Em geral, o controle no topo resultou em resistências coesivas menores do que o controle no CTOD (em média, 11\% menores). Já para a energia de fratura, foram observadas tendências diferentes para as temperaturas avaliadas. 


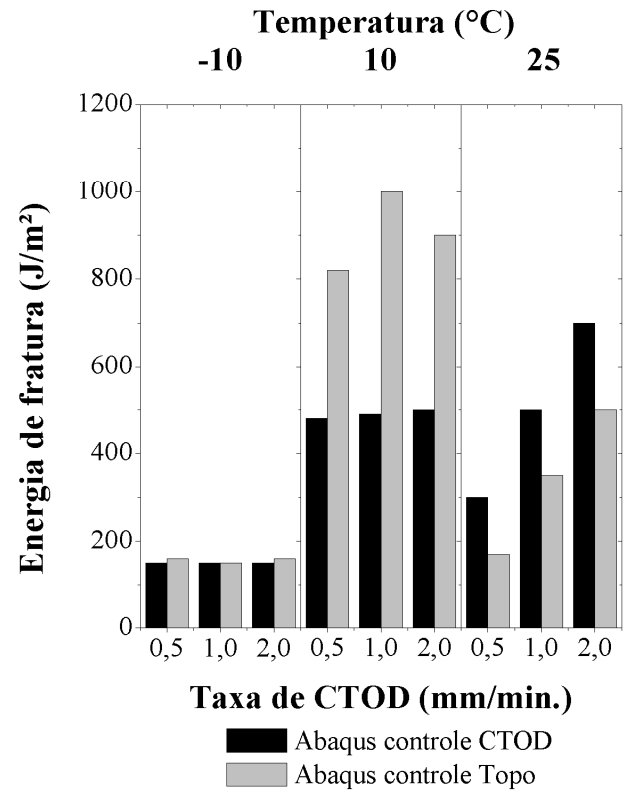

(a) Energia de fratura

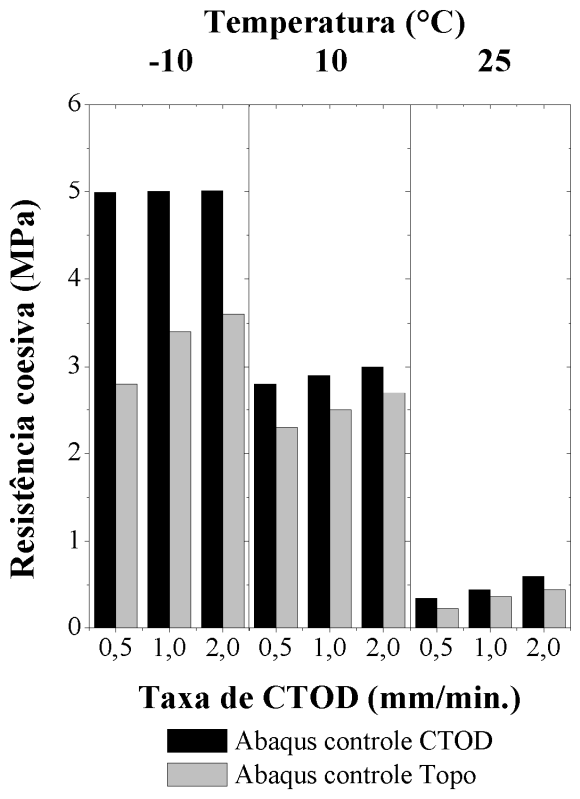

(b) Resistência coesiva

Figura 7: Comparação das propriedades de fratura obtidas com as duas formas de carregamento.

O controle do CTOD com uma função de deslocamento do topo subestimou os valores da resistência coesiva para todas as temperaturas $\left(-10^{\circ} \mathrm{C}, 10^{\circ} \mathrm{C}\right.$ e $\left.25^{\circ} \mathrm{C}\right)$ e taxas de abertura de CTOD avaliadas $(0,5 \mathrm{~mm} / \mathrm{min} ., 1,0 \mathrm{~mm} / \mathrm{min}$., $2,0 \mathrm{~mm} / \mathrm{min}$.). No caso da energia de fratura, o controle do CTOD como uma função de deslocamento do topo resultou em valores superestimados para as temperaturas de $-10^{\circ} \mathrm{C}$ e $10^{\circ} \mathrm{C}$ e subestimados para a temperatura de $25^{\circ} \mathrm{C}$, em todas as taxas de abertura de CTOD estudadas.

A Figura 8 mostra que o controle indireto da taxa de CTOD, a partir de uma função matemática imposta no atuador, não resultou em um valor constante para esta taxa. Por fim, a Figura 9 mostra que a taxa de abertura do CTOD é muito diferente para os dois tipos de controle. Assim, acredita-se que o controle direto da taxa de CTOD com o uso da sub-rotina implementada no trabalho é a opção mais apropriada, pois, diferente da opção pelo controle da taxa de descida do atuador, o controle no CTOD garante que a taxa de abertura da trinca seja próxima ao valor constante imposto em ensaios de carregamento monotônico. 


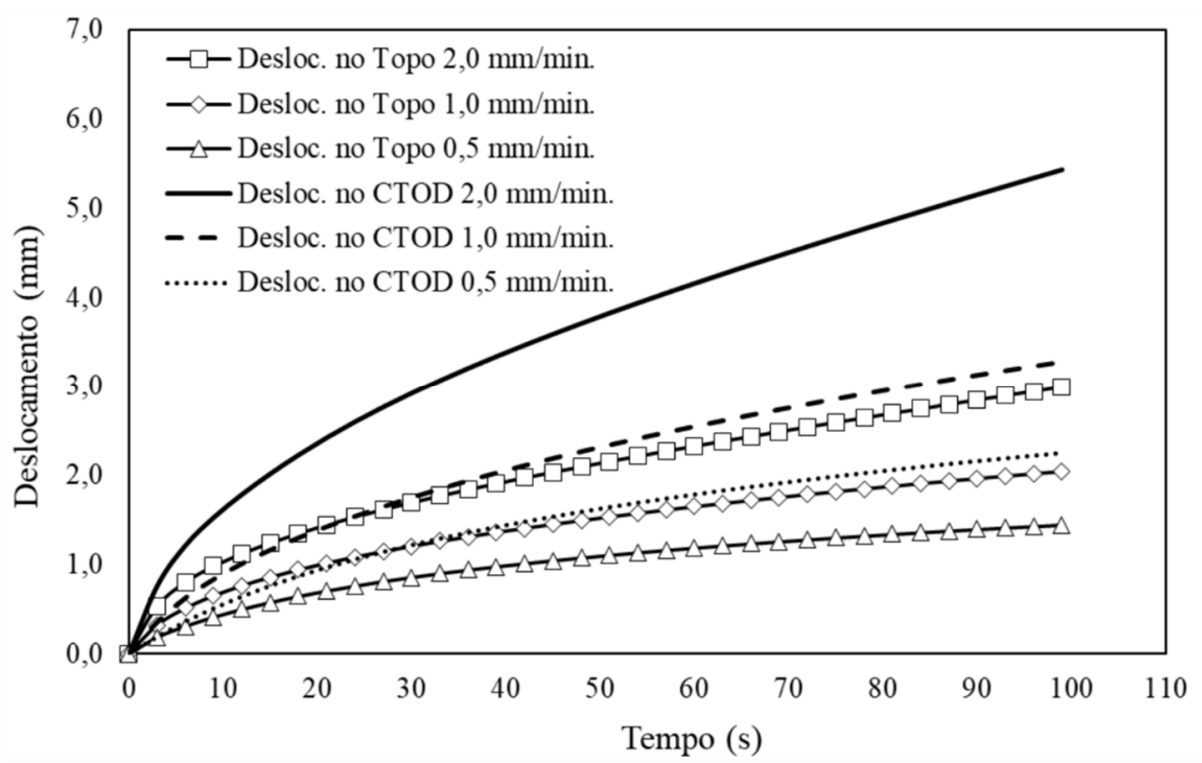

Figura 8: Variação dos deslocamentos do topo e da abertura do CTOD a $25^{\circ} \mathrm{C}$ para simulações realizadas com controle no topo por meio de uma função matemática.

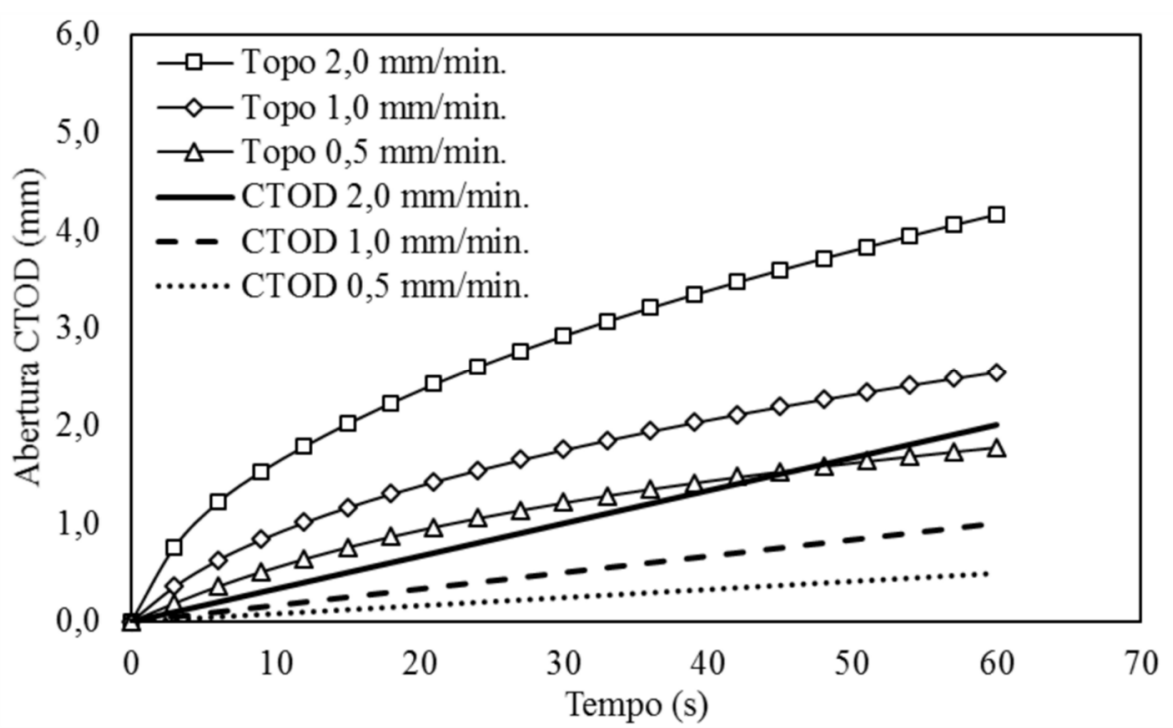

Figura 9: Variação da abertura do CTOD a 25C para simulações realizadas com controla na taxa de abertura do CTOD e controle na taxa de deslocamento do topo.

\section{CONCLUSÕES}

Este artigo avaliou três diferentes procedimentos de aplicação de carga em ensaios virtuais de uma MAF em modo I. Simulações numéricas de ensaios SCB conduzidos em laboratório foram incialmente realizadas com o carregamento aplicado no topo da amostra. A seguir, uma subrotina foi desenvolvida e incorporada ao software Abaqus para simular o processo iterativo conhecido como feedback loop de controle do movimento de descida do atuador em ensaios de laboratório de forma a garantir a aplicação de uma taxa constante de abertura da fenda inicial da amostra virtual. Nestas simulações, a evolução do deslocamento vertical do nó central, no topo da amostra, foi monitorada e modelada com uma função do tempo. Esta função foi usada em um terceiro tipo de controle do carregamento, aplicado no nó central do topo da amostra virtual, em simulações sem o uso da sub-rotina de controle da taxa de abertura na ponta da fenda inicial.

Os resultados obtidos no trabalho indicam que modelos numéricos desenvolvidos para as 
simulações de ensaios de fratura em modo I devem levar em consideração fatores como o modo de carregamento aplicado às amostras virtuais para garantir a representação fiel do processo de fratura de amostras reais ensaiadas em laboratório. 0 controle da taxa de abertura da fenda inicial parece ser uma opção mais apropriada do que os outros dois modos de controle, já que estes resultam em taxas variáveis de propagação das trincas.

Este trabalho faz parte de uma pesquisa em desenvolvimento pelos autores para a modelagem numérico-experimental do processo complexo de fratura de misturas asfálticas. Nas simulações, a definição adequada das condições de contorno é fundamental para permitir a obtenção de previsões mais realistas. Espera-se que a versão final do modelo sirva como uma ferramenta para o projeto e a análise de misturas asfálticas em que o comportamento global dos materiais poderá ser previsto a partir de características dos seus constituintes.

\section{AGRADECIMENTOS}

Os autores agradecem ao Conselho Nacional de Desenvolvimento Científico e Tecnológico (CNPq), à Coordenação de Aperfeiçoamento de Pessoal de Nível Superior (CAPES) e ao Laboratório Nacional de Materiais e Modelos Estruturais da Universidade da Costa Rica (LanammeUCR) pelo apoio financeiro recebido para a realização desta pesquisa.

\section{REFERÊNCIAS}

Aragão, F. T. S. e Kim, Y. R. (2012). Mode I fracture characterization of bituminous paving mixtures at intermediate service temperatures. Experimental Mechanics, v. 52, n. 9, p. 1423-1434. DOI: 10.1007/s11340-012-9594-4.

Aragão, F. T. S.; D. A. Hartmann; Y. R. Kim; L. M. G. Motta e M. Haft-Javaherian (2014). A numerical-experimental approach to characterize fracture properties of asphalt mixtures at low in-service temperatures. Transportation Research Record: Journal of the Transportation Research Board. Washington, D.C., n. 2447, p. 42-50. DOI: 10.3141/2447-05.

Budny, J. (2012). Avaliação do comportamento mecânico de misturas asfálticas mornas. Dissertação (Mestrado em Engenharia Civil). COPPE/UFRJ. Rio de Janeiro, Brasil.

Hirsch, F. (2009). Avaliação das propriedades de misturas asfálticas através do ensaio de tração em disco circular com fenda. (Mestrado em Engenharia Civil). COPPE/UFRJ. Rio de Janeiro, Brasil.

Li, X. e M. O. Marasteanu (2004). Evaluation of the low temperature fracture resistance of asphalt mixtures using the semi circular bend test. Journal of the Association of Asphalt Paving Technologists, v. 73, p. 401-426. ISSN: 0270-2932

$\mathrm{Li}, \mathrm{X}$ e M. O. Marasteanu (2010). Using semi circular bending test to evaluate low temperature fracture resistance for asphalt concrete. Experimental Mechanics, Inc., v. 50, p. 867-876. DOI: 10.1007/s11340-009-9303-0.

Marasteanu, M. O.; S. T. Dai; J. F. Labuz e X. Li (2002). Determining the low-temperature fracture toughness of asphalt mixtures. In Transportation Research Record: Journal of the Transportation Research Board, n. 1789, p. 191-199. DOI: 10.3141/1789-21.

Marasteanu, M. O.; W. Buttlar; H. Bahia e C. Williams (2012). Investigation of low temperature cracking in asphalt pavements, National Pooled Fund Study -Phase II. Final Report MN/RC 2012-23. Minnesota Department of Transportation. Minnesota, Estados Unidos.

Song, S.H.; G. H. Paulino e W. G. Buttlar (2006). A bilinear cohesive zone model tailored for fracture of asphalt concrete considering viscoelastic bulk material. J. of Engineering Fracture Mechanics, p. 2829-2847. DOI: 10.1016/j.engfracmech.2006.04.030.

Song, S. H.; M. P. Wagoner e G. H. Paulino (2008). $\delta 25$ Crack opening displacement parameter in cohesive zone models: experiments and simulations in asphalt concrete. Fatigue and Fracture of Engineering Materials \& Structures, n. 31, p. 850-856. DOI: 10.1111/j.1460-2695.2008.01272.x.

Sousa, P.; E. Kassen; E. Masad e D. Little (2013). New design method of fine aggregates mixtures and automated method for analysis of dynamic mechanical characterization data. Construction and Building Materials, n. 41, p. 216-223. DOI: 10.1016/j.conbuildmat.2012.11.038.

Wagoner, M. P.; W. G. Buttlar; G. H. Paulino e P. Blankenship (2005). Investigation of the fracture resistance of hot-mix asphalt concrete using a disk-shaped compact tension test. TRR, n. 1929, p. 183-192. DOI: 10.1177/0361198105192900122. 